\title{
List of Participants Conference on Differential Geometry and Partial Differential Equations Brasilia, May 24-28, 2010
}

- Aminov, Yuriy (Kharkov State U, Ukraine)

- Araújo, Kellcio (UnB, Brazil)

- Araújo, Pedro (UnB, Brazil)

- Barbosa, João Lucas Marques (UFC, Brazil)

- Barros, Abdênago Alves (UFC, Brazil)

- Barroso, Nilton (UnB, Brazil)

- Beals, Richard (Yale U, USA)

- Bernardini, Matheus (UnB, Brazil)

- Bessa, Gregorio Pacelli Feitosa (UFC, Brazil)

- Biliotti, Leonardo (U Di Parma, Italy)

- Brasil Junior, Aldir Chaves (UFC, Brazil)

- Bryant, Robert (MSRI e Duke U, USA)

- Carvalho, Leonardo (UERJ, Brazil)

- Carvalho, Tânia (UFU, Brazil)

- Cavalheiro, Adail de Castro (UnB, Brazil)

- Cezana, Miguel (UnB, Brazil)

- Chaves, Rosa Maria dos Santos Barreiro (USP, Brazil)

- Corro, Armando Vasquez (UFG, Brazil)

- Costa, Wagner (UFAL, Brazil)

- Dajczer, Marcos (IMPA, Brazil)

- De Carvalho, Fernando Soares (UFG, Brazil)

- De Lima, Levi (UFC, Brazil)

- De Oliveira, Hudson Pina (UnB, Brazil)

- Do Carmo, Manfredo Perdigão (IMPA, Brazil)

- Dorfmeister, Josef (TU München, Germany)

- Dos Santos, João Paulo (UnB, Brazil)

- Espirito-Santo, Nedir (UFRJ, Brazil)

- $\quad$ Ferreira, Walterson Pereira (UFG, Brazil) 
- Ferro, Marcelo (UnB, Brazil)

- Filho, Robson (UnB, Brazil)

- Fontenele, Francisco (UFF, Brazil)

- Galvão, Maria Elisa (IME - USP, Brazil)

- Gomes, Leonardo (UnB, Brazil)

- Gomes Neto, Verissimo (UnB, Brazil)

- Goulart, Claudiano (UEFS, Brazil)

- Grama, Lino (UNICAMP, Brazil)

- Hieda, Lidiane (UFG, Brazil)

- Ivey, Thomas A. (College of Charleston, USA)

- Javaloyes, Miguel Angel (U de Murcia, Spain)

- Jorge, Luquesio P. (UFC, Brazil)

- Junior, Rinaldo (IMECC - UNICAMP, Brazil)

- Kamran, Niky (McGill U, Canada)

- Lappicy, Phillipo (UnB, Brazil)

- Leite, Maria Luiza Soares (UFPE, Brazil)

- Lemes, Max Valério (UFG, Brazil)

- Lima, Levi Lopes (UFC, Brazil)

- $\quad$ Lira, Jorge Herbert Soares (UFC, Brazil)

- Lobos, Guillermo (UFSCar, Brazil)

- Martinez, Antonio (U de Granada, Spain)

- Martins, Alisson (UnB, Brazil)

- Martins, Rafael (UERJ, Brazil)

- Melo, Marcelo (UFC, Brazil)

- Melo, Marcos (UFC, Brazil)

- Mercuri, Francesco (UNICAMP, Brazil)

- Nascimento, Arlyson (IFAL, Brazil)

- Negreiros, Caio José Colletti (UNICAMP, Brazil)

- Nunes, Augusto Cesar (UnB, Brazil)

- Oliveira, Clarissa (UERJ, Brazil)

- Olver, Peter (U of Minnesota, USA)

- Piccione, Paolo (USP, Brazil)

- Pieterzack, Mauricio Donizetti (UFG, Brazil)

- Pina, Romildo da Silva (UFG, Brazil)

- Prado, Rafaela (UnB, Brazil)

- Rabelo, Mauro Luiz (UnB, Brazil)

- Reis, Matheus (UFU, Brazil)

- $\quad$ Reis, Rodrigo (Faculdade Albert Einstein, Brazil)

- Reyes, Enrique (U de Santiago de Chile)

- Rigas, Alcebíades (UNICAMP, Brazil)

- Ripoll, Jaime Bruck (UFRGS, Brazil)

- Riveros, Carlos (UnB, Brazil)

- Robles, Colleen (Texas A\&M U, USA) 
- Rodrigues, Luciana Maria Dias de Ávila (UnB, Brazil)

- Roitman, Pedro (UnB, Brazil)

- Rosenberg, Harold (IMPA, Brazil)

- Santos, Walcy (UFRJ, Brazil)

- Santos dos Reis, Hiuri Fellipe (UFG, Brazil)

- Silva, Jairo (UnB, Brazil)

- Silva, Rosângela Maria (UFG, Brazil)

- Silva, Tarcísio (UnB, Brazil)

- Simon, Udo (TU Berlin, Germany)

- Siqueira Ramos, Thiago Williams (UnB, Brazil)

- Soares, Bruno (UFF, Brazil)

- Solórzano Chávez, Newton Mayer (UFG, Brazil)

- Souza, Anyelle (UnB, Brazil)

- Souza, Flávio (IFG, Brazil)

- Souza, Marcelo Almeida (UFG, Brazil)

- Tojeiro, Ruy (UFSCAR, Brazil)

- Tomei, Carlos (PUC-RJ, Brazil)

- Tribuzy, Renato de Azevedo (UFAM, Brazil)

- Wang, Qiaoling (UnB, Brazil)

- Xavier, Frederico (U of Notre Dame, USA)

- Xia, Changyu (UnB, Brazil)

- Zhou, Detang (UFF, Brazil)

- Zhou, Jiazheng (UnB, Brazil) 